hep-th/0312194

\title{
A Matrix Model for $\mathrm{AdS}_{2}$
}

\author{
Andrew Strominger \\ Jefferson Physical Laboratory, Harvard University, Cambridge, MA 02138
}

\begin{abstract}
A matrix quantum mechanics with potential $V=\frac{q^{2}}{r^{2}}$ and an $\mathrm{SL}(2, \mathrm{R})$ conformal symmetry is conjectured to be dual to two-dimensional type $0 \mathrm{~A}$ string theory on $\mathrm{AdS}_{2}$ with $q$ units of RR flux.
\end{abstract}




\section{Contents}

1. Introduction . . . . . . . . . . . . . . . . . . . . . . . . . . . . . 1

2. Spacetime solutions of $c=10 \mathrm{~A}$ string theory . . . . . . . . . . . . . . . . 2

2.1. An extremal black hole . . . . . . . . . . . . . . . . . . . . . . . . . . . 2

2.2. Tachyon Hair . . . . . . . . . . . . . . . . . . . . . . . . . . . . . . .

3. Static solutions of the $0 \mathrm{~A}$ matrix model . . . . . . . . . . . . . . . . . . . . 6

4. The tachyon mass in $\mathrm{AdS}_{2}$. . . . . . . . . . . . . . . . . . . . . . . . . . . 8

5. Time in the matrix model . . . . . . . . . . . . . . . . . . . . . . . . . . . . 9

\section{Introduction}

The reinterpretation [1-4] of $c=1$ matrix models as holographic duals of type 0 string theories has revitalized two-dimensional string theories as useful toy models for understanding their higher-dimensional cousins. In this paper we will use this to motivate a conjecture for an $\mathrm{AdS}_{2} / \mathrm{CFT}_{1}$ correspondence, relating an $\mathrm{AdS}_{2}$ solution of the type $0 \mathrm{~A}$ effective action to a conformally invariant matrix quantum mechanics.

Type 0A string theory in two dimensions has a single scalar "tachyon" as well as non-dynamical RR two-form fluxes [4]. The low energy effective action with $q$ units of electric flux has extremal black hole-like solutions with a near-horizon $\mathrm{AdS}_{2}$ region [5,6]. The string coupling in the $\mathrm{AdS}_{2}$ region can be made arbitrarily weak by making $q$ large. We argue in section 2 that the extremal solutions comprise a one parameter family of solutions labelled by the thickness $\mu$ of the "tachyon hair". However in the $\mathrm{AdS}_{2}$ region the curvature is of order one in string units, so results from the effective action are at best suggestive. In this paper we shall simply assume the existence of a tree-level $0 \mathrm{~A} \mathrm{AdS}_{2}$ solution. 目

A holographic dual for the 2D 0A string theory has been proposed [4] as the quantum mechanics of free fermions moving in the potential

$$
V=-\frac{r^{2}}{4 \alpha^{\prime}}+\frac{q^{2}}{2 r^{2}}
$$

This has a one parameter family of static solutions labelled by the energy $\mu$ of the fermi surface. We propose to identify this parameter with the thickness of the tachyon hair of the extremal black holes in the spacetime picture.

1 Since the string coupling can be made arbitrarily weak, the existence of such a region is a question for the string worldsheet, potentially answerable by an analysis of RR CFTs. 
At very small $r$, the potential (1.1) is approximated by

$$
V=\frac{q^{2}}{2 r^{2}} \text {. }
$$

We conjecture in section 3 that the free fermion theory with potential (1.2) is dual to the purported $\mathrm{AdS}_{2}$ solution of $0 \mathrm{~A}$. The main piece of evidence for this is that (1.2) defines an $\mathrm{SL}(2, \mathrm{R})$ conformally invariant quantum mechanics which matches the $\mathrm{SL}(2, \mathrm{R})$ isometries of $\mathrm{AdS}_{2}$.

This conjecture relates a well-defined matrix model to a $0 \mathrm{~A}$ spacetime solution which (unfortunately) has not been constructed in the full tree-level string theory. Therefore it is difficult at this point to find evidence for the conjecture, beyond matching the symmetries. We do explain in section 4 how to use the matrix model to compute the mass of the tachyon in $\mathrm{AdS}_{2}$ which might, at some later date, be compared to a worldsheet computation.

If correct our proposal has conceptual implications for the nature of time in the matrix model (see also [7]). We argue in section 5 that the usual matrix model hamiltonian $H$ generates evolution with respect to Poincare time in $\mathrm{AdS}_{2}$. Evolution in global time is generated by $L_{0}=\frac{1}{2}(H+K)$, where $K$ is the generator of special conformal transformations. The spectrum of $H$ is continuous while that of $L_{0}$ is discrete. Hence different operators in the free fermion theory correspond to hamiltonians which evolve along different time slicings.

Past treatments of matrix models have largely rigidly invoked a single hamiltonian. This is antithetical to the spirit of gravity in which there are many time slicings and many hamiltonians. We expect that quite generally, as in the example of this paper, different operators in the various matrix models will have illuminating interpretations as different hamiltonians.

\section{Spacetime solutions of $c=10 \mathrm{~A}$ string theory}

\subsection{An extremal black hole}

The low-energy effective action of 0A string theory in two dimensions, with RR electric field proportional to $q$ and $\alpha^{\prime}=1$, is [4: 8$]^{2}$

$$
S_{e f f}=\int d^{2} x \sqrt{-g}\left(e^{-2 \Phi}\left(8+R+4(\nabla \Phi)^{2}-\frac{1}{2}(\nabla T)^{2}+T^{2}\right)-\frac{q^{2}}{2}-q^{2} T^{2}+\ldots . .\right) .
$$

2 The low energy theory contains two $U(1)$ gauge fields $F^{+}$and $F^{-}$which give rise to oppositesigned tachyon tadpoles. The action given here is for the sector of the theory in which both the electric flux in both sectors is proportional to $q$, and the tachyon tadpole is cancelled. $q$ is the net number of D0brane sources up to factors of 2 and $\pi$. 
Static, extremal black hole-like solutions with $T=0$ of this (and more general actions) have been found in [5, 6, 9]

$$
\begin{aligned}
d s^{2} & =\left(1+\frac{q^{2}}{8}\left(\Phi-\Phi_{0}-\frac{1}{2}\right) e^{2 \Phi}\right)\left(-d t^{2}+d \sigma^{2}\right) \\
\sigma(\Phi) & =\frac{1}{\sqrt{2}} \int^{\Phi} \frac{d \Phi^{\prime}}{1+\frac{q^{2}}{8}\left(\Phi^{\prime}-\Phi_{0}-\frac{1}{2}\right) e^{2 \Phi^{\prime}}} \\
\Phi_{0} & \equiv-\ln \frac{q}{4}
\end{aligned}
$$

In the asymptotic region $\sigma \rightarrow-\infty$ (or $\sigma<<-\ln q)$, the coupling becomes weak, the effect of the flux $q$ is diminished, and the solution approaches the usual linear dilaton vacuum

$$
\begin{array}{rlrl}
d s^{2} & \rightarrow-d t^{2}+d \sigma^{2}, & \\
\Phi & =\sqrt{2} \sigma, \quad \sigma \rightarrow-\infty .
\end{array}
$$

In the "near-horizon" region $\sigma \rightarrow+\infty(\sigma>>\ln q)$, $\Phi$ reaches the critical value $\Phi=\Phi_{0}$ and the conformal factor of the metric in (2.2) vanishes quadratically

$$
\begin{gathered}
d s^{2} \rightarrow-2\left(\Phi-\Phi_{0}\right)^{2}\left(d t^{2}-d \sigma^{2}\right) \\
\Phi \rightarrow \Phi_{0}-\frac{1}{2 \sqrt{2} \sigma} \quad \sigma \rightarrow+\infty
\end{gathered}
$$

The Killing vector $\partial_{t}$ becomes null, so we identify $\sigma=\infty$ as a horizon. The near-horizon geometry is

$$
\begin{array}{rlrl}
d s^{2} & =\frac{1}{4 \sigma^{2}}\left(-d t^{2}+d \sigma^{2}\right), & & \\
\Phi & =\Phi_{0} & \sigma \rightarrow+\infty .
\end{array}
$$

This is easily recognized as the $S L(2, R)$ invariant metric on the Poincare patch of $\mathrm{AdS}_{2}$. The string coupling $e^{\Phi_{0}}=\frac{4}{q}$ will be small if $q$ is large. 3 On the other hand the scalar curvature is

$$
R=-8, \quad \ell_{A d S}=\frac{1}{2}
$$

in string units. This means that, although string loops are suppressed by large $q, \alpha^{\prime}$ corrections remain large and (2.1) cannot be trusted. For example $R^{2}$ or $R^{2} T^{2}$ terms are important. Nevertheless we shall proceed by assuming that the suggestion of a nearhorizon $\mathrm{AdS}_{2}$ region is correct, and that (2.5) corresponds to an $\mathrm{AdS}_{2}$ solution of $0 \mathrm{~A}$ string

3 We note that this $q$-dependence agrees with a matrix model determination of the effective string coupling in [9]. 
theory at weak string coupling. In principle this might be verified from a study of string worldsheet CFTs with RR backgrounds, although with our current level of understanding of RR backgrounds this may not be an easy task.

The full solution (2.2) hence describes the two dimensional reduction of an extremal black hole interpolating from a flat linear dilaton region at infinity to an $\mathrm{AdS}_{2}$ near-horizon region. The present paper largely concerns the $\mathrm{AdS}_{2}$ region on its own. However there are infrared subtleties in the physics of $\mathrm{AdS}_{2}$ [10,11] which suggest that the decoupling of the near horizon and asymptotic regions may not be as simple as it is for the higher-dimensional analogs. Further progress in this area will likely require a better understanding of these issues.

There are also non-extremal analogs of the solutions (2.2) [6] which have a finite Hawking temperature and are quantum mechanically unstable. These solutions differ by an integration constant (the mass parameter) to the conformal factor in (2.2) and have a single rather than a double zero at the horizon. However the focus of this paper will be exactly static stable solutions and these non-extremal solutions will accordingly not be considered.

\subsection{Tachyon Hair}

When $q=0$, (2.2) reduces to the familiar linear dilaton vacuum. This solution has a one-parameter (usually denoted by $\mu$ ) family of static deformations. In the asymptotic region the solution has a nonzero tachyon field decaying as a certain linear combination of $\mu \sigma e^{\sigma}$ and $\mu e^{\sigma}$. In this subsection we discuss the generalization of this family of solutions to nonzero $q$.

The linearized tachyon equation of motion following from $S_{e f f}$ is

$$
\nabla^{2}\left(e^{-\Phi} T\right)+\left(2-(\nabla \Phi)^{2}+\nabla^{2} \Phi-\frac{q^{2}}{\pi} e^{2 \Phi)}\right)\left(e^{-\Phi} T\right)=0
$$

In the asymptotic region, for time-independent $T$ this becomes

$$
-\partial_{\sigma}^{2}\left(e^{-\sqrt{2} \sigma} T\right)=0
$$

4 Black hole no-hair theorems do not apply here because of the weak boundary conditions at infinity. 
which leads to the general asymptotic solution 5

$$
T \sim-(\mu \sigma+a) e^{\sqrt{2} \sigma}, \quad \sigma \rightarrow-\infty
$$

In the near-horizon $\mathrm{AdS}_{2}$ region the equation becomes

$$
\begin{gathered}
e^{-\Phi_{0}}\left(\nabla^{2} T-m_{T}^{2} T\right)=0, \\
m_{T}^{2}=30,
\end{gathered}
$$

whose general static solution is

$$
T \sim b_{-} \sigma^{h_{-}}+b_{+} \sigma^{h_{+}} \quad \sigma \rightarrow+\infty
$$

where [8]

$$
h_{ \pm}=\frac{1}{2}\left(1 \pm \sqrt{1+4 m_{T}^{2} \ell_{A d S}^{2}}\right)
$$

We expect the actual values of $h_{ \pm}$to be affected by $\alpha^{\prime}$ corrections, but we will assume that $h_{-}$is zero or negative. We will see below that the matrix model correspondence predicts definite values for $h_{ \pm}$. Knowledge of the exact solution of (2.7), which could be solved numerically, would relate the two parameters $(\mu, a)$ to $\left(b_{-}, b_{+}\right)$, but the numerical coefficient will not be needed for our purposes. Regularity on the horizon at $\sigma=\infty$ requires that $b_{+}=0$, which in turn fixes $a$ as a linear function of $\mu$.

To summarize, the action (2.2) has a one-parameter family of smooth, static, quantummechanically stable solutions which may be described as extremal black holes with tachyon hair. The parameter can be taken to be the coefficient $\mu$ of the exponential tail of the tachyon at spatial infinity. These solutions generalize the usual tachyon wall solutions to nonzero $q$.

5 We find it convenient in the current context to adopt conventions in which $\mu$ is a positive, rather than negative, multiple of the energy. 


\section{Static solutions of the $0 \mathrm{~A}$ matrix model}

Type $0 A$ theory with $D 0$-brane flux proportional to $q$ in two dimensions is described by the matrix quantum mechanics. This can be expressed as a theory of free fermions $\Psi(x)$ moving on the plane in the radially symmetric potential[国 ${ }^{6}$

$$
V=-\frac{r^{2}}{4}+\frac{q^{2}}{2 r^{2}}
$$

The fermions are constrained to have zero angular momentum. This theory has a one parameter family of solutions which can be simply described as filling all states in the fermi sea up to energy $\mu$. We conjecture that this parameter $\mu$ is (proportional to ) the coefficient $\mu$ parameterizing the tachyon hair of the previous section. 6 Let us try to see how this fits together for $q \neq 0$ in the 0 A theory.

For large $q$ the quantum mechanics has two dynamical regions, divided around $r^{2} \sim q$, where the potential is dominated by the $-r^{2}$ or $\frac{q^{2}}{r^{2}}$ terms. Deep in the first region we can ignore the $\frac{q^{2}}{r^{2}}$ term. Hence this can be identified as the linear dilaton region. In the second region $r^{2}<<q$ the free fermion hamiltonian is approximately

$$
H=\frac{1}{2} \int d^{2} x \Psi^{\dagger}\left(p^{2}+\frac{q^{2}}{r^{2}}\right) \Psi
$$

where $p^{2}=-\frac{1}{r} \partial_{r} r \partial_{r}$ on radially symmetric wavefunctions. It is natural to identify this as the $\mathrm{AdS}_{2}$ region $\mathrm{B}$ We conjecture that (3.2) on its own describes string theory in $\mathrm{AdS}_{2}$.

6 There is a potentially important subtlety here which we have not resolved. In [4] this potential is argued to be relevant to the case of only one type of zero brane charge, for which the spacetime effective action has a tachyon tadpole [8]. It is not clear in this case how the tadpole is cancelled: in the (not fully reliable) effective action (2.1) we cancel this tadpole by turning on the second charge. Therefore it is possible that an enlarged (due to the second type of Dobranes) matrix model could be relevant to the $\mathrm{AdS}_{2}$ spacetime solutions.

To be precise $q^{2}$ in the expressions of this section should be replaced by $q^{2}-\frac{1}{4}$ but this difference, as well as convention dependent numerical factors relating this $q$ to the one of the previous section, are unimportant here and will be suppressed.

7 The special case $\mu=0$ of this conjecture is due to the authors of $[9]$.

8 The precise relation between $r$ and the spacetime coordinate $\sigma$ could be complicated due to leg pole factors. Our identification of the matrix model and spacetime quantities will not require this relation, but will proceed (see below) via the $\mathrm{SL}(2, \mathrm{R})$ symmetries. 
An important check on this conjecture is provided by the symmetries. (3.2) turns out to be conformally invariant. The $S L(2, R)$ symmetry is generated by $H$ together with

$$
K=\frac{1}{2} \int d^{2} x \Psi^{\dagger} r^{2} \Psi \quad D=\frac{1}{2} \int d^{2} x \Psi^{\dagger}\left(r p_{r}+p_{r} r\right) \Psi
$$

where here $p_{r}=-i \partial_{r}$. The commutators are

$$
[D, H]=2 i H, \quad[D, K]=-2 i K, \quad[H, K]=-i D
$$

This $S L(2, R)$ matches the $S L(2, R)$ isometries of the gravity $\mathrm{AdS}_{2}$ solution, which are generated by, in the metric (2.5),

$$
H=i \partial_{t}, \quad D=-2 i\left(\sigma \partial_{\sigma}+t \partial_{t}\right), \quad K=i\left(2 t \sigma \partial_{\sigma}+t^{2} \partial_{t}+\sigma^{2} \partial_{t}\right)
$$

This is the main piece of evidence for the conjecture. If correct the duality relating the quantum mechanics described by (3.2) to $0 \mathrm{~A}$ string theory with $q$ units of flux in the geometry (2.5) is an example of an $A d S_{2} / C F T_{1}$ correspondence. 9

Next we consider the static solutions with fermi surfaces at energies $\mu$. These correspond to quantum states $|\mu\rangle$ obeying

$$
H|\mu>=\mu| \mu\rangle
$$

9 We wish to draw attention to a possibly relevant set of conformal quantum theories known as the Calogero-Moser models and considered in a related context in [7]. These are obtained by replacing the eigenvalue potential of the $\mathrm{AdS}_{2}$ matrix model

$$
V=\sum_{i=1}^{N} \frac{q^{2}}{\lambda_{i}^{2}}
$$

with

$$
V=\sum_{i<j}^{N} \frac{q^{2}}{\left(\lambda_{i}-\lambda_{j}\right)^{2}} .
$$

Similar quantum-mechanical models arise in the study of black hole moduli spaces [12]. (3.7) gives an integrable, conformally invariant quantum theory. Hence, as pointed out in [7], it is a natural candidate for the $C F T_{1}$ dual to an $\mathrm{AdS}_{2}$ string theory. In this context it is intriguing to note that exactly this theory arises in the non-singlet sector of the $c=1$ matrix model [13]. This may be the proper setting for the spacetime dual to the Calogero-Moser sought in [7]. 
For large negative $\mu$, a small wave coming in from spatial infinity on the fermi sea is reflected long before it reaches the $\mathrm{AdS}_{2}$ region. In the gravity picture this is because large negative (with our conventions) $\mu$ corresponds to a large positive tachyon wall shielding the $\mathrm{AdS}_{2}$ region. On the other hand for large positive $\mu$ the wave will travel deep into the $\mathrm{AdS}_{2}$ region before being reflected. This is because positive $\mu$ corresponds to negative tachyon field which goes to zero at spatial infinity and the $\mathrm{AdS}_{2}$ horizon, creating a tachyon ditch which sucks the waves across the potential barriers separating the linear dilaton and $\mathrm{AdS}_{2}$ regions. These potential barriers are the same as those that lead to greybody factors in black hole evaporation. For large $\mu$ and deep in the $\mathrm{AdS}_{2}$ region the fermi surface obeys

$$
p^{2}+\frac{q^{2}}{r^{2}}=\mu
$$

The nonzero eigenvalue for $H$ corresponds to a spontaneous breaking of the SL(2,R) symmetry.

\section{The tachyon mass in $\mathbf{A d S}_{2}$}

Due to the lamentable current status of our technology for understanding RR worldsheet CFTs, we do not know if there is a $\mathrm{AdS}_{2}$ worldsheet CFT for the $0 A$ theory, let alone how to compute the spectrum. This makes it difficult to find any checks of our conjecture. However, assuming the conjecture, we can make a prediction for the tachyon mass (in units of the $\mathrm{AdS}_{2}$ curvature) in this CFT as follows.

The dilation operator $D$ rescales the hamiltonian according to

$$
e^{-i \alpha D} H e^{i \alpha D}=e^{2 \alpha} H \text {. }
$$

Hence

$$
e^{i \alpha D}|\mu\rangle=\left|e^{2 \alpha} \mu\right\rangle
$$

On the other hand in the $\mathrm{AdS}_{2}$ solution the dilation operator is the Killing vector

$$
D=-2 i \sigma \partial_{\sigma}-2 i t \partial_{t}
$$

This acts on the near horizon tachyon field (2.12) (with $b_{+}=0$ ) as

$$
e^{-i \alpha D} T=e^{-2 \alpha h_{-}} T
$$


Recalling that $T$ is itself proportional to $\mu$ this is equivalent to

$$
\mu \rightarrow e^{-2 \alpha h_{-}} \mu
$$

Comparing (4.5) to (4.2) leads to

$$
h_{-}=-1 \text {, }
$$

or equivalently a tachyon mass

$$
m_{T}^{2}=\frac{2}{\ell_{A d S}^{2}},
$$

where $\ell_{A d S}$ is the $\mathrm{AdS}_{2}$ radius. This may be viewed as a prediction for the spectrum of the worldsheet RR CFT .

\section{Time in the matrix model}

The conjectured $\mathrm{AdS}_{2} / \mathrm{CFT}_{1}$ correspondence has implications for the nature of time in the matrix model. The following discussion parallels that given in a related context by Gibbons and Townsend [7].

The hamiltonian $H$ in (3.2) generates time translations $i \partial_{t}$ in the Poincare patch of $\mathrm{AdS}_{2}$ with metric

$$
d s^{2}=\frac{-d t^{2}+d \sigma^{2}}{4 \sigma^{2}} .
$$

This patch covers only a wedge-shaped region of $\mathrm{AdS}_{2}$. Global coordinates can be defined by

$$
\tau \pm w=2 \arctan (t \pm \sigma)
$$

in terms of which the metric is

$$
d s^{2}=\frac{-d \tau^{2}+d w^{2}}{4 \sin ^{2} w}
$$

Translation in global time $\tau$ are generated by

$$
L_{0} \equiv i \partial_{\tau}=\frac{1}{2}(H+K)
$$

Identifying the spacetime Killing vectors (3.5) with the matrix model operators (3.2), (3.3), $L_{0}$ corresponds to a matrix model hamiltonian

$$
L_{0}=\frac{1}{2} \int d x \Psi^{\dagger}\left(p^{2}+\frac{q^{2}}{r^{2}}+r^{2}\right) \Psi
$$


Unlike $H, L_{0}$ has a potential which grows in both directions and a corresponding discrete spectrum. This is in agreement with the expected discrete spectrum of string theory in $\mathrm{AdS}_{2}$. The $S L(2, R)$ invariant ground state is the state with no fermions in the coordinates (5.3). $L_{0}$ is naturally written as part of a $\mathrm{SL}(2, \mathrm{R})$ multiplet

$$
L_{0}=\frac{1}{2}(H+K), \quad L_{ \pm 1}=\frac{1}{2}(H-K \mp i D)
$$

obeying

$$
\left[L_{0}, L_{ \pm 1}\right]=\mp L_{ \pm 1}, \quad\left[L_{1}, L_{-1}\right]=2 L_{0} .
$$

It is natural to characterize the $\mathrm{AdS}_{2}$ matrix model in terms of $L_{0}$ rather then $H$ eigenstates. In the gravity picture, this simply corresponds to a different choice of time .

There is potentially a general lesson for matrix models here. In the spacetime picture, they are theories of gravity. Theories of gravity have many equivalent descriptions corresponding to different time slicings and different hamiltonians. Often it is necessary to consider a variety of time slicings to fully understand the physics. The usual description of matrix models involves a single fixed hamiltonian $H$. Our current perspective suggests that some operators other than the usual $H$ in the matrix model should correspond to different hamiltonians and different time slicings.

\section{Acknowledgements}

This work was supported in part by DOE grant DE-FG02-91ER40654. I am grateful to J. Maldacena, S. Minwalla, J. Polchinski, N. Seiberg, S. Shenker, D. Thompson, X. Yin and especially J. Karczmarek and T. Takayanagi for useful conversations. 


\section{References}

[1] J. McGreevy and H. Verlinde, "Strings from tachyons: The $\mathrm{c}=1$ matrix reloaded," arXiv:hep-th/0304224.

[2] I. R. Klebanov, J. Maldacena and N. Seiberg, "D-brane decay in two-dimensional string theory," JHEP 0307, 045 (2003) arXiv:hep-th/0305159.

[3] T. Takayanagi and N. Toumbas, "A matrix model dual of type 0B string theory in two dimensions," JHEP 0307, 064 (2003) arXiv:hep-th/0307083.

[4] M. R. Douglas, I. R. Klebanov, D. Kutasov, J. Maldacena, E. Martinec and N. Seiberg, "A new hat for the $\mathrm{c}=1$ matrix model," arXiv:hep-th/0307195.

[5] T. Banks and M. O'Loughlin, "Nonsingular Lagrangians for two-dimensional black holes," Phys. Rev. D 48, 698 (1993) arXiv:hep-th/9212136.

[6] N. Berkovits, S. Gukov and B. C. Vallilo, "Superstrings in 2D backgrounds with R-R flux and new extremal black holes," Nucl. Phys. B 614, 195 (2001) arXiv:hepth/0107140].

[7] G. W. Gibbons and P. K. Townsend, "Black holes and Calogero models," Phys. Lett. B 454, 187 (1999) arXiv:hep-th/9812034.

[8] D. Thompson," AdS2 Solutions of 2D Type OA" hep-th/0312156.

[9] S. Gukov, T. Takayanagi and N. Toumbas, to appear.

[10] J. M. Maldacena, J. Michelson and A. Strominger, "Anti-de Sitter fragmentation," JHEP 9902, 011 (1999) arXiv:hep-th/9812073.

[11] M. Spradlin and A. Strominger, "Vacuum states for AdS(2) black holes," JHEP 9911, 021 (1999) arXiv:hep-th/9904143.

[12] R. Britto-Pacumio, J. Michelson, A. Strominger and A. Volovich, "Lectures on superconformal quantum mechanics and multi-black hole moduli spaces," arXiv:hepth/9911066.

[13] D. Boulatov and V. Kazakov, "One-dimensional string theory with vortices as the upside down matrix oscillator," Int. J. Mod. Phys. A 8, 809 (1993) arXiv:hepth/0012228]. 